\title{
CUIDADO DOMICILIAR: OBSTÁCULOS VIVENCIADOS POR CUIDADORAS DE PESSOAS COM MOBILIDADE FÍSICA PREJUDICADA
}

\section{HOME CARE: OBSTACLES EXPERIENCED BY CAREGIVERS OF PERSONSWITH IMPAIRED PHYSICAL MOBILITY}

Renata Karoline Farias Silva ${ }^{1}$ Michelly Guedes de Oliveira Araújo ${ }^{2}$ Michelinne OliveiraMachado Dutra ${ }^{3}$

Rosilene Santos Baptista ${ }^{4}$

RESUMO: Objetivo: identificar as possíveis dificuldades sentidas por cuidadoras informais de pessoas com mobilidade física prejudicada, bem como orientá-las visando qualificar o cuidado prestado. Método: estudo descritivo com abordagem qualitativa, realizado no período de fevereiro a maio de 2016, por meio de visita domiciliar, em Unidades Básicas de Saúde do município de Campina Grande Paraíba. Os sujeitos do estudo foram mulheres cuidadoras de pessoas com mobilidade física prejudicada. A coleta foi realizada em três momentos: aplicação de questionário para traçar o perfil das cuidadoras; observação, através da qual se detectou as necessidades apresentadas e qualificação das cuidadoras na prestação dos cuidados. $\mathrm{Na}$ análise dos dados, utilizou-se a técnica de análise de conteúdo, da qual surgiram três grandes categorias: Limitações das cuidadoras x prática do cuidar, Dificuldades no ato de cuidar e Sentimentos das cuidadoras x Pessoa cuidada. Resultados: Em relação aos dados obtidos, verificou-se que: são inúmeras as limitações enfrentadas pelas cuidadoras, devido à falta de orientação e apoio por parte dos profissionais de saúde; as dificuldades presentes no exercício do cuidar geram consequências para quem está sendo cuidado e para a própria cuidadora; a orientação oferecida às cuidadoras informais favorece a prestação de uma assistência qualificada. Conclusão: essa pesquisa foi de grande contribuição para desenvolver nas cuidadoras uma visão crítica e a partir deste ponto, potencializar suas ações de cuidado, colaborando para aquisição de conhecimento e habilidades

\footnotetext{
${ }^{1}$ Enfermeira pela UEPB.

2 Enfermeira. Doutoranda em Enfermagem pela UPE/UEPB. Docente do curso de Enfermagem do UNI-RN.

3 Enfermeira. Doutora em Enfermagem pela UPE/UEPB. Docente do curso de Medicina da UNIFACISA.

${ }^{4}$ Enfermeira. Doutora em Enfermagem pela UFC. Docente do curso de Enfermagem UEPB.
} 
que proporcionam um cuidado direcionado a patologia do cliente, possibilitando a prestação de uma assistência qualificada.

Palavras chave: Assistência domiciliar. Cuidadores. Enfermagem.

ABSTRACT: Objective: to identify the possible difficulties experienced by informal caregivers of people with impaired physical mobility, as well as to guide them in order to qualify the care provided. Method: descriptive study with a qualitative approach, conducted from February to May 2016, through home visits in Unidades Básicas de Saúde (Basic Health Units) in the city of Campina Grande - Paraíba. The study subjects were women caregivers of people with impaired physical mobility. The collection was carried out in three moments: application of a questionnaire to outline the caregivers' profile; observation, through which the needs presented were detected; and qualification of the caregivers in the provision of care. In the data analysis, the content analysis technique was used, from which three major categories emerged: Caregivers' limitations $x$ care practice, Difficulties in the act of caring, and Caregivers' feelings $x$ person cared for. Results: In relation to the data obtained, it was verified that: there are countless limitations faced by the caregivers due to the lack ofguidance and support from health professionals; the difficulties present in the practice of caring generate consequences for those being cared for and for the caregiver herself; the guidance offered to informal caregivers favors the provision of a qualified assistance. Conclusion: This research was of great contribution to develop a critical view in the caregivers and, from this point, to enhance their care actions, collaborating to the acquisition of knowledge and skills that provide a care directed to the client's pathology, enabling the provision of a qualified assistance.

Keywords: Home Nursing. Caregivers. Nursing. 


\section{INTRODUÇÃO}

O cuidar é mais que uma ação, é uma atitude, que pode representar um envolvimento afetivo, preocupação e responsabilização com o outro. Perpassa toda a existência humana, pois é inerente a sua própria condição de sobrevivência. A prática do cuidado é desenvolvida desde as primeiras civilizações do Oriente e Ocidente, ressaltando-se não só nos velhos países do continente europeu, como também nas culturas orientais (ROCHA; VIEIRA; SENA, 2008;GEOVANINI, 2002).

A principal meta do cuidar é restabelecer e promover a saúde. Neste sentido existem duas modalidades de atenção de maior visibilidade no Brasil, a hospitalar e a de atenção básica. Substitutiva ou complementar a essas modelagens, o cuidado no domicílio vem ganhando espaço. Porém ressalta-se que antes mesmo do surgimento dos hospitais e ambulatórios, já se praticava a atenção domiciliar, por meio da qual algumas pessoas eram favorecidas com uma assistência individualizada, enquanto outra camada da população ficava dependente de hospitais filantrópicos e curandeiros (BRASIL, 2012).

Desse modo, quando se fala em cuidar remete-se na maioria das vezes à figura feminina. Isso se deve ao fato do papel de estabelecer o cuidado ser designado predominantemente à mulher, pois ela cuida do lar, dos filhos e de tudo que envolve o toque, a delicadeza, o carinho. Sabendo-se que frequentemente esta sobrecarga de tarefas e responsabilidades requer uma maior atenção, é imprescindível que na atenção domiciliar se tenha um olhar mais direcionado por parte da equipe de saúde, atentando para os aspectos emocionais e o domínio das técnicas utilizadas para estabelecer a assistência. É importante uma capacitação voltada para estas mulheres/cuidadoras, de forma a empoderá-las para que estas possam desenvolver suas habilidades pessoais de acordo com os recursos que the são disponíveis (ARAÚJO et al, 2013; ARAÚJO et al, 2015).

A equipe de saúde, em especial o enfermeiro, precisa analisar estratégias para uma efetivação do cuidado, vislumbrando o aspecto psicológico, físico e 
emocional, inserindo o cuidador como peça fundamental neste processo. Deste modo, deve-se trabalhar as potencialidades das cuidadoras desenvolvendo junto a elas medidas que venham somar na assistência prestada e favorecer resultados significativos para o paciente. A cuidadora deve serorientada a fim de desenvolver atividades que promovam não só a sua saúde mental e física, mas também a saúde do cliente assistido por ela (CAETANO, 2012; OLIVEIRA et al., 2014).

Diante de tais considerações, se faz necessário alguns questionamentos: De que modo às mulheres cuidadoras sem formação na área de saúde, estão prestando esta assistência no domicílio? Estas foram orientadas para tal? Portanto, realizou-se esta pesquisa com o objetivo de responder a tais indagações e identificar as possíveis dificuldades que as cuidadoras enfrentam no seu cotidiano; assim como orientar cuidadoras informais de pessoas com mobilidade física prejudicada numa perspectiva de qualificar o cuidado prestado.

\section{MÉTODO}

Trata-se de um estudo descritivo com abordagem qualitativa e integrativa, realizado no período de fevereiro a maio de 2016, na cidade de Campina Grande PB. Os sujeitos da pesquisa foram mulheres cuidadoras de pessoas com mobilidade física prejudicada. A coleta de dados foi realizada no próprio domicílio das cuidadoras, com o intuito de presenciar a rotina de cuidados. Foram escolhidos de forma aleatória três distritos de Campina Grande, sendo eles: o distrito II, III e IV. Após essa escolha, realizou-se um levantamento do quantitativo de pacientes com mobilidade física prejudicada nas unidades que compõem os distritos. As unidades básicas de saúde com o maior número de cuidadoras informais de pacientes com mobilidade prejudicada foram João Rique I e II do distrito II, Monte Santo do distrito III e João Benoni de Andrade do distrito IV. As participantes foram selecionadas seguindo os critérios de inclusão e exclusão, juntamente com os agentes comunitários de saúde (ACS's) de cada território. Inicialmente 32 cuidadoras foram 
convidadas a participarem da pesquisa, porém a amostra final totalizou 19 cuidadoras.

Foram incluídas mulheres que atenderam aos seguintes critérios: ter idade igual ou superior a 18 anos, prestar cuidado no período igual ou superior a dois meses, ser cuidadoras de pacientes com mobilidade prejudicada, aceitar participar da pesquisa e ter paciente cadastrado em uma das Unidade Básica de Saúde da Família - UBSF de referência para o projeto no município de Campina Grande - PB. Foram excluídas as mulheres que: prestavam cuidados no período inferior a dois meses; não concordaram em participar da pesquisa e cuidadoras de pessoas com mobilidade física adequada.

A coleta foi realizada em três momentos: aplicação de questionário para traçar perfil sociodemográfico das cuidadoras, a realização de uma observação, através da qual se detectou as necessidades apresentadas e a qualificação das cuidadoras na prestação dos cuidados.

Inicialmente foi feito um contato com as enfermeiras das UBSF's, logo em seguida, um levantamento do quantitativo de pessoas com mobilidade prejudicada em cada área junto aos Agentes Comunitários de Saúde (ACS's), que foram o elo entre os pesquisadores e os sujeitos da pesquisa. No segundo instante realizou-se o primeiro contato com as cuidadoras do domicílio, juntamente com os ACS's, explicando o projeto e solicitando a assinatura do Termo de Consentimento Livre e Esclarecido (TCLE). Após concordarem participar da pesquisa, foi aplicado o questionário que traçou o perfil sociodemográfico das cuidadoras. Logo após realizou-se a observação com o intuito de conhecer os cuidados prestados à pessoa com mobilidade física prejudicada, para finalmente orientar essas cuidadoras nas ações prestadas. Essas orientações foram realizadas no domicílio, de acordo com as dificuldades de cada cuidadora e foram baseadas em técnicas de semiotécnica e nos manuais do ministério da saúde voltados a cuidadores informais.

A análise e discussão dos dados foram feitas mediante categorização do material coletado através do questionário aplicado, da observação participante e da orientação realizada, baseados na análise de conteúdo e apoiado nos recursos literários pertinentes ao tema fundamentado nos conceitos de promoção da saúde. 
Esta pesquisa foi desenvolvida de acordo com os parâmetros da Resolução 466/12 do Conselho Nacional de Saúde e Ministério da Saúde, que dispõem sobre a pesquisa envolvendo seres humanos. O termo de Consentimento Livre e Esclarecido foi assinado pelas participantes, e todos foram informados sobre os objetivos da pesquisa, o sigilo e a não identificação como participante (BRASIL, 2012a). O presente estudo foi aprovado no comitê de ética em pesquisa da Universidade Estadual da Paraíba, sob o número CAAE: 53324116.1.0000.5187.

Com o intuito de manter o sigilo e preservar o anonimato das cuidadoras participantes da pesquisa, determinou-se um cognome para cada mulher. Todas as cuidadoras são mencionadas através da letra alusiva $C$ seguida de um número, que foi colocado na ficha e no questionário para facilitar a identificação para a pesquisadora.

\section{RESULTADOS}

Inicialmente a amostra foi composta por 32 mulheres cuidadoras de pacientes com mobilidade física prejudicada. Porém, após algumas visitas, descobriu-se que dois dos pacientes haviam falecidos. Além disso, por motivos pessoais, cinco cuidadoras se recusaram a continuar participando da pesquisa, e, após uma conversa com outras três, observou-se que os respectivos pacientes não apresentavam mobilidade física prejudicada a ponto de depender de cuidados, sendo essas excluídas da coleta. Por fim, eliminou-se também três casos que representaram riscos para a pesquisadora, resultando assim num total de 19 cuidadoras participantes da pesquisa.

Percebe-se que descrever o perfil do cuidador e ter conhecimento das dificuldades que porventura estes vivenciam no dia a dia, se torna essencial para a implementação de ações educativas e de suporte social, pois desta forma, consegue-se trabalhar com a realidade que o cuidador está inserido. Nesse contexto, o papel de cuidador informal está muito ligado a um membro da família, na maioria das vezes a mulher, seja ela esposa, filha, neta ou mãe. 
O presente estudo teve como foco as cuidadoras, tendo em vista que os achados de outras pesquisas mostram que as mulheres ainda dominam o cenário do cuidado, mesmo trabalhando fora ou exercendo outras atividades dentro do âmbito domiciliar. Participaram da coleta 19 mulheres, com idade entre 36 a 76 anos, com uma média de aproximadamente 58,15 anos. Segundo o grau de parentesco das cuidadoras, notou-se que a maioria são esposas e filhas nas mesmas proporções com 36,84\% ( $n=7)$ cada, 15,78\% ( $n=3)$ são mães, 5,26\% ( $n=1)$ irmãs e 5,26\% $(n=1)$ noras, esse resultado é semelhante a outros estudos. Araújo et al. (2015), revela que $38,4 \%$ dos cuidadores precipitantes de sua pesquisa eram filhos e afirma que o parentesco é um fator que influencia muito no momento de escolher um cuidador.

Quanto ao grau de escolaridade, observou-se que a maioria das mulheres tinha o ensino fundamental incompleto, somando $47,36 \%$ ( $n=9)$ no total, $10,52 \%$ ( $n=$ 2) possuíam ensino fundamental completo, $10,52 \%(n=2)$ o ensino médio incompleto, $26,31 \%(n=5)$ haviam completado o ensino médio e $5,2 \%(n=1)$ não tinham escolaridade alguma.

Em seu estudo, Azevedo (2011) mostra que há uma distinção na forma de cuidar de acordo com o grau de escolaridade da cuidadora, onde as semianalfabetas e de ensino fundamental incompleto apresentam mais dificuldade na compreensão do tratamento.

Com relação à situação conjugal das cuidadoras, pode-se observar que a maioria delas, está envolvida em relacionamentos estáveis e $90 \%$ possuem filhos. Segundo Araújo et al. (2013) esses dados refletem na sobrecarga que essas mulheres possuem, pois além do papel de cuidadora, elas carregam as responsabilidades de esposa, de mãe, de ter que cuidar da casa, delavar a roupa e de atender aos chamados dos filhos e marido. $O$ relato a seguir contribui com essa análise:

"Tem hora que falta pouco pra eu ficar louca, eu tenho que dar o banho dele, tenho que preparar o café de todo mundo, meu marido me chama o tempo todo." (C5) 
Ainda para Araújo et al (2013), a situação conjugal no cenário do cuidar, pode ser tanto positiva, se o marido consegue participar de forma ativa, como também pode ser bastante negativa para a cuidadora, se o marido não ajuda e ainda a sobrecarrega com a cobrança diária das atividades domesticas.

De acordo com os resultados extraídos do questionário, da observação participante e da orientação realizada junto às cuidadoras, foi possível obter três categorias temáticas: 1. Limitações das cuidadoras $\mathrm{x}$ prática do cuidar; 2. Dificuldades no ato do cuidar e 3. Sentimentos das cuidadoras x pessoa cuidada.

\section{Limitações das cuidadoras $x$ prática do cuidado}

Esta pesquisa teve como principal objeto de estudo cuidadoras informais, que segundo Bailão (2013, p.6):

\footnotetext{
"Refere-se ao familiar ou amigo, que assegura a maior parte dos cuidados que o doente requer quando regressa ao seu contexto familiar, assumindo-se, de modo não remunerado, como principal responsável pela assistência e prestação de cuidados à pessoa doente."
}

Diante do exposto, pode-se dizer que, cuidadoras informais constituem o apoio principal de cuidado, sem receber nenhuma remuneração e sem ter preparo técnico, ou seja, nenhuma formação na área de saúde, para a realização das atividades assistenciais necessárias. Araújo et al. (2015).

No estudo de Oliveira et al. (2014), ressalta-se que as próprias cuidadoras percebem a necessidade de estarem sendo orientadas por um profissional da saúde, que compreenda de forma mais efetiva as técnicas importantes para o fortalecimento de um cuidado eficaz, justamente porque o conhecimento que elas possuem não é suficiente para a adequada execução das atividades do cuidado e para a prevenção de possíveis complicações. 
As limitações técnicas no desenvolvimento do cuidado provocam nas cuidadoras informais sentimento de angústia. Elas se sentem despreparadas para desempenharem algumas atividades necessárias ao cuidado, e inseguras quanto à forma correta de se realizar os procedimentos (OLIVEIRA et al., 2012).

"No começo foi uma barra muito grande que enfrentei, não queria aceitar de jeito nenhum. A primeira vez que eu tive que passar a sondapra retirar o xixi dele, eu pensei: $\mathrm{Eu}$ não vou conseguir, nunca fiz isso antes, eu vou morrer." (C16)

"Quando o médico colocou a traqueo nele, e me disse que eu ia terque aspirar, fiquei com medo, nunca tinha feito nada parecido, nunca cuidei de ninguém, mas pelo meu filho eu tive que enfrentar." (C17) "Tento fazer o melhor que eu posso, não sei se faço tudo certo, mas sempre procuro aprender porque nunca cuidei de ninguém na minhavida." (C2)

No relato abaixo, observa-se o fato de que, quanto maior a limitação física do paciente domiciliar, maior a dificuldade por parte das cuidadoras, frente aos cuidados. Isso ocorre, devido à complexidade e a quantidade das atividades que um paciente com um grau elevado de imobilidade necessita, requerendo do cuidador um maior suporte técnico, como também uma boa condição física.

"Ah minha filha, tudo desse homem é nessa cama, se banha na cama, come na cama, e é difícil de lidar, ele é pesado e eu não consigo pegar sozinha. A fralda nunca fica direito, ele acaba urinando o lençol todinho." (C7)

O vínculo familiar existente entre cuidadora-paciente revela, em alguns casos, uma relação conflituosa, devido ao tipo de relacionamento que existia anteriormente. Este é um fator que também limita o cuidado. Observou-se no discurso de algumas 
filhas que havia constrangimento no momento de cuidar, pois o pai sempre preservou uma posição de autoridade com os filhos.

"Na hora do banho, ele não quer que eu fique com ele de jeito nenhum, tenho que chamar minha mãe pra ajudar ele. $\mathrm{E}$ eu prefiro, pois meu pai sempre foi um homem muito sério e reservado." (C8)

Visto que a falta de orientação para o ato de cuidar acarreta no cuidador informal uma sobrecarga, isso culmina na geração de desgastes físicos e emocionais (VIEIRA et al., 2011). Diante disso, percebe-se a importância do acompanhamento e da orientação por parte de um profissional da área de saúde no desenvolvimento do cuidado domiciliar, de modo a minimizar a diversidade de sentimentos que surgem no decorrer do exercício, como também, suprir as limitações técnicas do cuidado, visto que estas acarretam prejuízos tanto à saúde da pessoa cuidada, como a do próprio cuidador.

\section{Dificuldades no ato do cuidar}

O estudo exacerbou que 13 cuidadoras relataram não ter dificuldade para realizar o cuidado. Percebe-se com os discursos abaixo que, na visão das cuidadoras, a habilidade e até mesmo a melhor compreensão sobre a realização de ações que envolvem o cuidado ao paciente domiciliar, foram adquiridas através do tempo de experiência, da rotina e do empenho em realizar o melhor.

"Não sinto dificuldade não, eu faço o melhor que posso. E também jásão 6 anos fazendo isso todos os dias." (C16) "Cuidei da minha mãe durante muito tempo, agora cuido do meu pai, aprendi muita coisa nesse tempo, tenho uma experiência boa." (C10) "Ah faz muitos anos que cuido 
dele, já sei como tudo funciona. Não são dias, não, são anos e anos, fazendo a mesma coisa, todo santodia."(C5)

Cuidar é um ato que envolve conhecimento, habilidade e até mesmo valores, que quando colocados em prática favorecem e potencializam a melhora da condição de vida. No cotidiano das cuidadoras informais, existem diversos desafios, como as dificuldades em realizar as atividades complexas do cuidado, sem nenhum suporte técnico (VIEIRA et al., 2011).

Constata-se que a maioria das cuidadoras subestimam a capacidade do paciente em realizar algumas atividades do seu próprio cuidado. Este fato torna-se um grande problema no contexto domiciliar, pois o cuidador acaba anulando a autonomia do paciente, exercendo sobre ele uma proteção excessiva, que na verdade mais prejudica do que contribui para a sua melhora. De acordo com Araújo et al. (2015), o medo e a insegurança tornam o paciente fragilizado, de modo que este acaba aceitando de forma passiva os cuidados excessivos ofertados pelos cuidadores. Este problema é evidenciado nas falas abaixo:

"Eu faço tudo, até a comida dou na boca, por que fico imaginando que pra ela deve ser muito difícil, e também se fosse deixar ela comer sozinha ia demorar demais. Quando ela quer água, eu também dou na boca dela, e também se não faço isso o marido dela, diz que eu sou ruim." (C3)

Nos relatos pode-se perceber que, diante das dificuldades, as cuidadoras acabamagindo de forma mais afetiva do que racional, o que prejudica a reabilitação do paciente.

Diante desse problema, alertou-se as cuidadoras para a importância da estimulação da autonomia do paciente, que é essencial para a evolução clínica do mesmo. Estudos mostram que a participação dos pacientes nas atividades do cotidiano, voltadas para o próprio cuidado, trazem benefícios de melhora da 
funcionalidade, evitando que estes adquiram ainda mais limitações, além de diminuir a sobrecarga da própria cuidadora (ARAÚJO et al., 2015).

Em relação aos principais problemas em comum, observados entre as cuidadoras, está a dificuldade no exercício das atividades que exigem um maior esforço físico, como a locomoção, a realização do banho no leito, a transferência da cadeira para cama e vice-versa, a troca de roupa e de fralda. Como também está na realização de procedimentos que necessitam de conhecimento e habilidades técnicas: troca de curativos, prevenção de úlceras por pressão, realização da higiene íntima, administração da medicação e passagem de sonda vesical.

O banho no leito é uma atividade desempenhada com grande dificuldade, por grande parte das cuidadoras. Se torna ainda mais difícil de ser realizado em virtude da falta de condições adequadas dentro do âmbito domiciliar, onde as camas são baixas e os espaços pequenos, e, devido a esses fatores, realizar o banho exige da cuidadora um maior esforço físico e uma maior habilidade, como descrito nas falas:

"Eu não consigo dar banho nele sozinha, não. Só passo um paninho molhado, pra tirar a catinga da fralda." (C7)

"O banho é o pior momento, por que ele é pesado demais, e minha coluna não aguenta. Fora que eu acabo molhando tudo, pra secar ele todo é uma briga, acho que jogo muita água." (C5)

Neste sentido, foram ofertadas instruções básicas para a realização do banho no leito, através das quais, as cuidadoras foram direcionadas a seguirem uma ordem necessária, como forma de organizar e facilitar seu trabalho, como também de tornar o período do banho agradável para a pessoa cuidada. Iniciando sempre pela cabeça, e seguindo para os braços, tórax, abdômen, lavando e já secando, evitando a exposição do paciente. As cuidadoras foram orientadas a no momento do banho, massagear a pele do acamado para ativar a circulação, bem como observar a sua integridade, os cabelos e as unhas. (POTTER; PERRY; ELKIN, 2009; BRASIL, 2010).

Uma outra dificuldade presente no contexto do cuidado domiciliar analisada, foi a prevenção das úlceras por pressão. Considerando que os pacientes 
participantes apresentavam mobilidade física prejudicada como critério de inclusão na pesquisa, a necessidade de medidas preventivas contra o aparecimento dessas feridas se tornavam essenciais ao cuidado, tendo em vista, que a maioria era acamado ou cadeirante, o que são fatores de risco para o surgimento das úlceras.

Por consequência da falta de medidas preventivas, alguns dos pacientes já apresentavam as feridas, e daí surge uma outra dificuldade para as cuidadoras, que é a falta de habilidade para execução dos curativos, descritos nos relatos:

"Vou botar cloro, já cuidei de outras feridas e sempre fica bom quando boto o cloro. Já não sei mais o que fazer para essas feridas nãoaparecerem." (C7)

Diante disso, foram passadas algumas orientações para as cuidadoras, com o intuito de direcioná-las. No primeiro momento, a ênfase foi na prevenção do aparecimento das úlceras. Visto que a ingesta hídrica e a nutrição também constituem medidas preventivas para o aparecimento das úlceras por pressão, se fez necessário alertar as cuidadoras para a importância da oferta de líquidos e da manutenção de alimentação balanceada (BRASIL, 2010; BRASIL, 2013; MORAES et al., 2013).

A higienização das mãos é uma medida primordial para a realização dos cuidados. No domicílio notou-se que esta é uma ação, na maioria das vezes, esquecida e deixada de lado por parte das cuidadoras na hora de executarem os cuidados.

Portanto, foram ofertadas orientações voltadas para a importância da lavagem das mãos e a forma correta de se fazer. Priorizando o fato de que essa é uma medida de segurança, que visa a proteção do paciente, e por isso deve ser feita sempre antes e após o contato com o mesmo (BRASIL, 2010).

Percebe-se que, quando as dificuldades das cuidadoras são supridas, o cuidado se tornamais leve e eficaz. A qualificação das cuidadoras reflete no cuidado que elas prestam, e consequentemente na qualidade de vida da pessoa cuidada. (CAETANO, 2012). 


\section{Sentimentos das cuidadoras $\mathrm{x}$ pessoa cuidada}

A ação de cuidar requer um conceito amplo que envolve além de técnica, vertentes afetiva, racional, sociocultural, ética e terapêutica. Diante disso, percebese a complexidade da palavra "cuidar", pois ela envolve um olhar holístico que englobe todas as necessidades do ser cuidado (SARAIVA, 2011).

O cuidar provoca uma diversidade de sentimentos na cuidadora, dentre eles alguns positivos e outros negativos. De fato, isto ocorre pois existem laços afetivos que acompanham a relação familiar, que por vezes geram no cuidador gratidão, satisfação em poder cuidar, vontade de estar ao lado do ente querido o máximo de tempo possível, mas também tristeza por ver a situação de dependência da pessoa cuidada, medo, falta de paciência (OLIVEIRA et al, 2014). As mulheres quando indagadas a respeito de como se sentiam em relação à pessoa cuidada, transmitiram os seguintes discursos:

"Me sinto triste, pois lembro de como ela era antes, e ver ela hoje nessa situação, me deixa muito triste. Fico pensando como tudo poderia ter sido diferente, ela casada, dando as aulas que tanto gostava, com filho." (C12)

"Sem paciência, pois ele quer tudo do jeito dele, se não for na hora que ele pede, é por que eu trato ele mal, que depois que ele ficou aleijado, ninguém mais respeita." (C13)

As cuidadoras demonstram, em suas falas, a sensação de impotência diante do estado de saúde do seu familiar e da impossibilidade de transformar a situação. Este fato acarreta um desgaste emocional, que é percebido através de sentimentos negativos como tristeza, inquietação, preocupação. 
Percebe-se que a cuidadora informal vivência de forma muito intensa as alterações apresentadas pelo paciente como a agitação, a agressividade, as diversas reclamações, atristeza e a baixa autoestima. Esse misto de sentimentos é consequência do estado de dependência que se encontra decorrente da própria patologia (PINTO; NATIONS, 2012). Percebe-se que a cuidadora acaba se expondo a vários fatores estressantes, como: o acúmulo de atividades, a diversidade de sentimentos expressados pela pessoa cuidada, além disso, a falta de informação e de apoio, como descrito nas falas:

"Ele reclama é de tudo, depois que ele ficou assim, só reclama! Parece até que eu não faço nada que preste. Falta é a paciência tem hora." (C13)

"Cansada, minha filha, muito cansada! Estou é carregando a cruz que Deus me deu." (C7)

Diante dos relatos, observa-se que esta sobrecarga emocional pode limitar a tolerância das cuidadoras perante algumas situações do cotidiano, refletindo em conflitos, insatisfações e frequentemente em diversos sentimentos negativos que surgem durante o exercício da complexa atividade de cuidar. Nota-se que a maneira como a pessoa que necessita de cuidados trata o cuidador, pode revelar o fato da não aceitação da sua situação de dependência.

A família costuma centralizar todas as responsabilidades do cuidado em uma única pessoa, tornando-a cuidadora exclusiva e esquecendo e até subestimando as necessidades pessoais que esta possui. A falta de apoio formal fornecido por parte de profissionais da saúde, e de apoio informal, fornecido por outros membros da família e amigos, é motivo para o acúmulo de sobrecarga nas cuidadoras, e para o surgimento de sentimentos negativos que interferem diretamente no cuidado que é prestado. 


\section{CONCLUSÃO}

Os trabalhos e pesquisas com abordagem nos cuidadores são escassos. Tal fato reflete no desconhecimento sobre o papel que estes indivíduos desenvolvem e sobre a necessidade de apoio que eles requerem no desempenho de atividades diárias do cuidado. A falta de conhecimento em torno deste assunto gera consequências, sendo uma delas a grande proporção de limitações encontradas no cuidado dentro do contexto domiciliar.

Neste sentido, o tema da pesquisa é de grande relevância, tendo em vista que possibilitou um olhar mais dedicado para as cuidadoras informais, as quais enfrentam em seu cotidiano diversas limitações na prestação do cuidado de pacientes com mobilidade prejudicada, devido à falta de apoio e orientação por parte dos profissionais de saúde.

Vale ressaltar que a enfermagem tem papel fundamental no que diz respeito ao apoio as cuidadoras diante das dificuldades evidenciadas por estas no cotidiano. O direcionamento do cuidado oferece uma assistência mais efetiva $e$ consequentemente uma melhor qualidade de vida para a pessoa cuidada.

A orientação oferecida por meio desta pesquisa foi de grande contribuição para desenvolver nas cuidadoras uma visão crítica e a partir deste ponto, potencializar suas ações de cuidado. Colaborando para aquisição de conhecimento e habilidades que proporcionam um cuidado mais direcionado a patologia do paciente, possibilitou-se a prestação de uma assistência mais qualificada.

\section{REFERÊNCIAS BIBLIOGRÁFICAS}

ARAÚJO, J. B. et al. Efeitos de uma capacitação oferecida a cuidadores informais de pacientes pós-AVC. Revista Neurociências, São Paulo, v. 23, n. 3, p. 368-375, 2015.

ARAUJO, J. S. et al. Perfil dos cuidadores e as dificuldades enfrentadas no cuidado ao idoso, em Ananindeua, PA. Rev. bras. geriatr. gerontol., Rio de Janeiro, v. 16, n. 1, p.149-158, 2013.

AZEVEDO, M. F.M. et al. Perfil do conhecimento de cuidadores de pacientes pediátricos sobre 
medicamentos prescritos. Rev. Ciênc. Farm. Básica Apl. Araraquara, v. 32, n. 2, p. 245-249, 2011.

BAILÃO, A.L.S. O cuidador informal em contexto oncológico: Sobrecarga e qualidade de vida familiar. 2013. $91 \mathrm{f}$. Dissertação (Mestrado em Psicologia) Faculdade de Psicologia e de Ciências da Educação. Coimbra - PT.

BRASIL. Conselho Nacional de Saúde. Resolução n 466, de 12 de dezembro de 2012a. Dispõe sobre diretrizes e normas regulamentadoras de pesquisas envolvendo seres humanos. Diário Oficial da União: seção 1, Brasília, DF, ano 150, n. 112, p. 59-62, 13 jun. 2013.

BRASIL. Instituto nacional do câncer (INCA). Guia do cuidador de pacientes acamados: orientações aos pacientes. $2^{\mathrm{a}}$. ed. Rio de Janeiro, 2010.

BRASIL. Ministério da saúde. Secretaria de atenção à saúde. Departamento de atenção básica e Coordenação-geral de atenção domiciliar. Caderno de atenção domiciliar, Vol.1 - BrasíliaDF, 2012.

BRASIL. Secretaria de atenção à saúde. Departamento de atenção básica e Coordenação-geral de atenção domiciliar. Caderno de atenção domiciliar, Vol.2 - Brasília-DF, 2013.

CAETANO, F.T.B. Perfil do cuidador domiciliar de idoso no município de Taquaritinga- SP. 82 f. Dissertação (Mestrado em Saúde na Comunidade) - Faculdade de Medicina de Ribeirão Preto/USP, Ribeirão Preto - SP, 2012.

MORAES, G. L. A. et al. Aplicação de protocolo de prevenção de úlcera por pressão no contexto domiciliar: uma trajetória percorrida. Cogitare Enferm., Curitiba, v. 18, n. 2, p. 387391, 2013.

OLIVEIRA, M.C. et al. Percepção do cuidador familiar de idosos dependentes sobre o papel do profissional da saúde em sua atividade. Semina: Ciências Biológicas e da Saúde, Londrina, v. 35, n. 2, p. 81-90, 2014.

OLIVEIRA, W. T. et al. Vivência do cuidador familiar na prática do cuidado domiciliar ao doente crônico dependente. Ciênc. Cuid. Saúde, Maringá, v. 11, n. 1, p. 129-137, 2012.

PINTO, Juliana Maria de Sousa and NATIONS, Marilyn Kay.Cuidado e doença crônica: Cuidado e doença crônica: visão do cuidador familiar no Nordeste brasileiro. Ciênc. saúdecoletiva. 2012, vol.17, n.2, pp. 521-530.

POTTER, P. A; PERRY. A; ELKIN, M.K. Fundamentos da enfermagem. ed.7. Rio de Janeiro: Elsevier, 2009.

ROCHA, M. P. F.; VIEIRA, M. A.; SENA, R. R. Desvelando o cotidiano dos cuidadores informais de idosos. Rev. Bras. Enferm., Brasília, v. 61, n. 6, p. 801-808, 2008.

SARAIVA, D. M. F. O olhar dos e pelos cuidadores: Os impactos de cuidar e a importância do apoio ao cuidador. 2011. 135 f. Tese (Mestrado em Intervenção Social) - Universidade de Coimbra, Coimbra - PT.

VIEIRA, C. P. B. et al. Práticas do cuidador informal do idoso no domicílio. Revista Brasileira de Enfermagem, Brasília, v. 64, n. 3, p. 570-579, 2011. 